\title{
TIME LAGS AND DENSITY DEPENDENCE IN AGE DEPENDENT TWO SPECIES COMPETITION
}

\author{
K. Gopalsamy
}

\begin{abstract}
Sufficient conditions are obtained for the existence and linear stability of time independent age distributions in two species competition with age and time lagged density dependent mortality and fertility functions.
\end{abstract}

\section{Introduction}

Let $P_{1}(t)$ and $P_{2}(t)$ denote the total population sizes (or biomasses) at time $t \geq 0$ of two interacting species living in a common habitat and competing for a common pool of resources. The competition for resources will be implicit in our model similar to that in the two species Lotka-Volterra competition system. Assuming constant sex ratios in the two species we can consider $P_{1}(t)$ and $P_{2}(t)$ to be the population of females only; immigration, emigration and internal dispersion in the habitat are assumed to play no significant role in the dynamics of the community.

We suppose that the two species contain respectively $\rho_{1}(a, t) d a$ and $\rho_{2}(a, t) d a$ individuals with ages between $a$ and $a+d a(a \geq 0)$ at time $t$ so that we have

Received 13 October 1981. 
(1.1) $\quad P_{1}(t)=\int_{0}^{\infty} \rho_{1}(a, t) d a, p_{2}(t)=\int_{0}^{\infty} \rho_{2}(a, t) d a, t \geq 0$.

The rates of change of the two species densities are by definition $D \rho_{1}(\alpha, t)$ and $D \rho_{2}(a, t)$ where

$$
D \rho_{i}(a, t)=\lim _{h \rightarrow 0} \frac{\rho_{i}(a+h, t+h)-\rho_{i}(a, t)}{h}, i=1,2
$$

Assuming the existence of the limits in (1.2) we consider the following time lagged model system

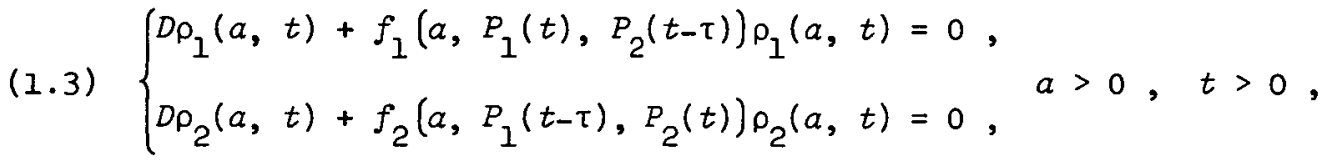

(1.4) $\begin{cases}\rho_{1}(0, t)=\int_{0}^{\infty} b_{1}\left(a, P_{1}(t), P_{2}(t-\tau)\right) \rho_{1}(a, t) d a, & \\ \rho_{2}(0, t)=\int_{0}^{\infty} b_{2}\left(a, P_{1}(t-\tau), P_{2}(t)\right) \rho_{2}(a, t) d a, & t>0,\end{cases}$

(1.5) $\rho_{i}(a, s)=\varphi_{i}(a, s), a>0, s \in[-\tau, 0], i=1,2$,

$$
P_{i}(s)=\int_{0}^{\infty} \varphi_{i}(a, s) d a, s \in[-\tau, 0]
$$

where $\tau$ is some fixed nonnegative constant; $f_{1}$ and $f_{2}$ denote the age and density dependent mortality rates with time lags in interspecific interactions, $b_{1}$ and $b_{2}$ denote the age and density dependent fertility functions again having time lags in the interspecific interactions; $\varphi_{1}$ and $\varphi_{2}$ denote the initial age distributions needed for the formulation of the model.

The model system (1.1)-(1.6) will be meaningful only if $\varphi_{1}, \varphi_{2}, f_{1}$, $f_{2}, b_{1}, b_{2}$ are nonnegative functions of the respective arguments. Also since the total initial populations have to be finite, $\varphi_{i}(\cdot, s)$ should belong to $L_{1}\left(R^{+}\right)$for each $s \in[-\tau, 0]$. We have specifically assumed 
that $f_{i}$ and $b_{i}(i=1,2)$ are independent of $t$ explicitly and this assumption can be interpreted to represent the temporal uniformity of the environmení .

One of the fundamental questions for $(1.1)-(1.6)$ is the following; under what conditions on $f_{i}, b_{i}$ and $\tau$ does the system (1.1)-(1.4) have time independent nontrivial solutions, and when such solutions exist, are they stable with respect to some suitable stability criterion? When such stable time invariant solutions exist for $(1.1)-(1.4)$ we say that the two species community has a stable age distribution. Existence of a stable age distribution is the analogue of the existence of a stable steady state for the corresponding age independent system.

In this article we investigate the above question for the case of a competitive interaction with some additional constraints on the vital rates $f_{i}$ and $b_{i}(i=1,2)$. Age dependent population systems without time delays have been considered by several authors (Gurtin and MacCamy [3, 4, 5], Gurtin and Levine [6], Haimovici [7] and Rotenberg [8]). The question of the relation between an age independent system and a corresponding age dependent system has been considered by Gurtin and MacCamy [4] and Gurtin and Levine [6] who have established an asymptotic (as $t \rightarrow \infty$ ) relation between such models by constructing a higher dimensional lumped parameter system (in terms of ordinary differential equations) to represent the age dependent distributed parameter system. Assuming the existence of stationary age distributions Haimovici [7] considers their stability in a system of two interacting populations explicitly taking into consideration the dynamical nature of the habitat's resources and pollution.

In this article we add another realistic feature namely time lags in the age dependent models and obtain sufficient conditions for the existence of stationary age distributions in (1.1)-(1.4) and show that the time lags in interspecific interactions have no effect on the linear stability of the age distributions although the decay rates of perturbations will depend on the magnitudes of the time delays. Such "harmless" nature of time lags in interspecific interactions have been noted by the author for the age independent models in $[1,2]$. 


\section{Existence and uniqueness of solutions}

We first narrate our assumptions on the vital rates of $f_{i}$ and $b_{i}$ $(i=1,2)$ so that the system (1.1)-(1.6) will denote a competition system. Let $B C[0, \infty)$ denote the linear space of bounded continuous real functions on $[0, \infty)$ with the norm defined by

$$
\|f\|=\{\max |f(t)|, t \in[0, \infty)\} \text {. }
$$

Let $K_{1}$ be the cone of nonnegative functions of $B C[0, \infty)$. Let $C[-\tau, 0]$ denote the space of real continuous functions with a norm defined by $\|g\|=\{\max |g(s)|, s \in[-\tau, 0]\}$; let $K_{2}$ be the nonnegative cone of functions in $C[-\tau, 0]$. We present our first set of assumptions on $f_{i}, b_{i}$ and $\varphi_{i}$ for $i=1,2$.

A . $f_{1} \in C\left(\mathbf{R}^{+} \times K_{1} \times K_{2}, \mathbf{R}^{+}\right), f_{1} \geq d_{1}^{*}$ on $\mathbf{R}^{+} \times K_{1} \times K_{2}$, $f_{2} \in C\left(\mathbf{R}^{+} \times K_{2} \times K_{1}, \mathbf{R}^{+}\right), f_{2} \geq d_{2}^{*}$ on $\mathbf{R}^{+} \times K_{2} \times K_{1}$, $\left(d_{1}^{*}, d_{2}^{*}\right.$ are positive constants);

$f_{1}$ and $f_{2}$ are Lipschitzian with respect to their last two arguments uniformly in $a \in \mathbf{R}^{+}$; that is, for $i=1,2$,

$$
\begin{aligned}
& \quad\left|f_{i}\left(a, P_{1}^{\prime}, P_{2}^{\prime}\right)-f_{i}\left(a, P_{1}^{\prime \prime}, P_{2}^{\prime \prime}\right)\right| \leq d_{i}\left[\left\|P_{1}^{\prime}-P_{1}^{\prime \prime}\right\|+\left\|P_{2}^{\prime}-P_{2}^{\prime \prime}\right\|\right] \\
& \left(d_{1}, d_{2} \text { are positive constants }\right),
\end{aligned}
$$

$$
\begin{aligned}
& \partial f_{1} / \partial P_{1}, \partial f_{1} / \partial P_{2} \in C\left(\mathbf{R}^{+} \times K_{1} \times K_{2}, \mathbf{R}^{+}\right), \\
& \partial f_{2} / \partial P_{1}, \partial f_{2} / \partial P_{2} \in C\left(\mathbf{R}^{+} \times K_{2} \times K_{1}, \mathbf{R}^{+}\right) .
\end{aligned}
$$

A. . $b_{1} \in C\left(\mathbf{R}^{+} \times K_{1} \times K_{2}, \mathbf{R}^{+}\right), b_{1} \leq \beta_{1}^{*}$ on $\mathbf{R}^{+} \times K_{1} \times K_{2}$, $b_{2} \in C\left(\mathbf{R}^{+} \times K_{2} \times K_{1}, \mathbf{R}^{+}\right), b_{2} \leq \beta_{2}^{*}$ on $\mathbf{R}^{+} \times K_{2} \times K_{1}$, $\left(B_{1}^{*}, B_{2}^{*}\right.$ are positive constants);

$b_{1}, b_{2}$ are Lipschitzian with respect to their last two arguments uniformly in $a \in \mathbf{R}^{+}$; that is, for $i=1,2$, $\left|b_{i}\left(a, P_{1}^{\prime}, P_{2}^{\prime}\right)-b_{i}\left(a, P_{1}^{\prime \prime}, P_{2}^{\prime \prime}\right)\right| \leq \beta_{i}\left[\left\|P_{1}^{\prime}-P_{1}^{\prime \prime}\right\|+\left\|P_{2}^{\prime}-P_{2}^{\prime \prime}\right\|\right]$ 


$$
\begin{aligned}
& \left(\beta_{1}, B_{2} \text { are positive constants }\right), \\
& b_{i}\left(\cdot, P_{1}, P_{2}\right) \in L^{\infty}\left(\mathbf{R}^{+}, \mathbf{R}^{+}\right) \text {for fixed } P_{1}, P_{2}, i=1,2, \\
& \quad \frac{\partial b_{1}}{\partial P_{2}}, \frac{\partial b_{1}}{\partial P_{2}} \in C\left(\mathbf{R}^{+} \times K_{1} \times K_{2}, \mathbf{R}^{-}\right), \\
& \\
& \quad \frac{\partial b_{2}}{\partial P_{1}}, \frac{\partial b_{2}}{\partial P_{2}} \in C\left(\mathbf{R}^{+} \times K_{2} \times K_{1}, \mathbf{R}^{-}\right) .
\end{aligned}
$$

A3. $\varphi_{i}(\cdot, s) \in L^{\perp}\left(\mathbf{R}^{+}, \mathbf{R}^{+}\right) \cap C\left(\mathbf{R}^{+}, \mathbf{R}^{+}\right)$for each $s \in[-\tau, 0]$, $i=1,2$.

By a solution of $(1.1)-(1.6)$ on $[0, T]$ we mean $\rho_{i}: \mathbf{R}^{+} \times[-\tau, T] \rightarrow \mathbf{R}^{+} \quad(i=1,2)$ with the following properties:

S1. $\rho_{i}(\cdot, t) \in L^{1}\left(\mathbf{R}^{+}, \mathbf{R}^{+}\right)$for each $t \in[-\tau, T]$;

S2. $\rho_{i}(a, \cdot) \in C\left([-\tau, T], \mathbf{R}^{+}\right)$for each $a \in \mathbf{R}^{+}$;

$S_{3} . D \rho_{i}$ exists along the characteristics $t-a=$ constant on $\mathbf{R}^{+} \times\left\{\mathbf{R}^{+} \cap[0, T]\right\}$ and is continuous $(i=1,2)$;

$S_{4} . \rho_{i}$ satisfies the system $(1.1)-(1.6)$ for $(a, t) \in \mathbf{R}^{+} \times[-\tau, T]$.

Let us first convert the system (1.1)-(1.6) into an equivalent system of integral equations. For brevity we denote by $P$ the pair $\left(P_{1}, P_{2}\right)$. If we let

$$
\rho_{i}(a+x, t+x)=\tilde{\rho}_{i}(x), i=1,2,
$$

then (1.3) considered at $(a+x, t+x)$ becomes

$$
\frac{d \tilde{\rho}_{i}(x)}{d x}+f_{i}(a+x, P(t+x)) \tilde{\rho}_{i}(x)=0
$$

which has a solution of the form

$$
\tilde{\rho}_{i}(x)=\tilde{\rho}_{i}(0) \exp \left[-\int_{0}^{x} f_{i}(a+s, P(t+s)) d s\right]
$$

and hence 
(2.1)

$$
\rho_{i}(a+x, t+x)=\rho_{i}(a, t) \exp \left[-\int_{0}^{x} f_{i}(a+s, P(t+s)) d s\right] .
$$

For $a \geq t$ we choose $(a, t)=(a-t, 0)$ in (1.3) and $x=t$ in (2.1) so that

$$
\begin{array}{r}
\rho_{i}(a, t)=\varphi_{i}(a-t, 0) \exp \left[-\int_{0}^{t} f_{i}\left(a-t+s, P_{i}(s)\right) d s\right], \\
0 \leq t \leq a, i=1,2,
\end{array}
$$

with

$$
\begin{aligned}
& P_{1}(s)=\left(P_{1}(s), P_{2}(s-\tau)\right), \\
& P_{2}(s)=\left(P_{1}(s-\tau), P_{2}(s)\right) .
\end{aligned}
$$

For $0 \leq a<t$ we choose $(a, t)=(0, t-a)$ in $(1.3)$ and $x=a$ in (2.1) so that

$$
\begin{array}{r}
\rho_{i}(a, t)=B_{i}(t-a) \exp \left[-\int_{0}^{a} f_{i}\left(s, P_{i}(t-a+s)\right) d s\right], \\
t>a \geq 0, i=1,2,
\end{array}
$$

where

$$
B_{i}(t)=\rho_{i}(0, t), \quad i=1,2, t>0 .
$$

Define $M_{i}$ and $L_{i} \quad(i=1,2)$ as follows:

$$
\begin{aligned}
& M_{i}\left(a, t, \mathbf{P}_{i}(t)\right)=\exp \left[-\int_{0}^{t-a} f_{i}\left(s, P_{i}(a+s)\right) d s\right], \\
& L_{i}\left(a, t, \mathbf{P}_{i}(t)\right)=\exp \left[-\int_{0}^{t} f_{i}\left(a+s, \mathbf{P}_{i}(s)\right) d s\right] .
\end{aligned}
$$

It will now follow from $(1.2),(1.3),(2.2)-(2.5)$ that

$$
\text { (2.6) } \begin{array}{r}
P_{i}(t)=\int_{0}^{t} B_{i}(a) M_{i}\left(a, t, \mathbf{P}_{i}(t)\right) d a+\int_{0}^{\infty} \varphi_{i}(a, 0) L_{i}\left(a, t, \mathbf{P}_{i}(t)\right) d a, \\
t>0, i=1,2,
\end{array}
$$

(2.7) $P_{i}(s)=\Phi_{i}(s)=\int_{0}^{\infty} \varphi_{i}(a, s) d a, s \in[-\tau, 0], i=1,2$, 
(2.8) $B_{i}(t)=\int_{0}^{t} B_{i}(a) b_{i}\left(t-a, \mathbb{P}_{i}(t)\right) M_{i}\left(a, t, \mathbb{P}_{i}(t)\right) d a$

$$
\begin{array}{r}
+\int_{0}^{\infty} b_{i}\left(a+t, \mathbf{P}_{i}(t)\right) \varphi_{i}(a, 0) L_{i}\left(a, t, \mathbf{P}_{i}(t)\right) d a, \\
i=1,2, t>0 .
\end{array}
$$

The equivalence of $(2.6)-(2.8)$ with $(1.1)-(1.6)$ is established by the following whose proof is identical to a similar result of Gurtin and MacCamy [4]; hence we omit the details of the proof of the following.

THEOREM 1. Suppose the system (1.1)-(1.6) satisfies the conditions $\mathrm{A}_{1}, \mathrm{~A}_{2}, \mathrm{~A}_{3}$. If $\left(\rho_{1}, \rho_{2}\right)$ is a solution of $(1.1)-(1.6)$ then the total populations $P_{1}, P_{2}$ and the birth rates $B_{1}, B_{2}$ satisfy the integral equations (2.6)-(2.8). Conversely if $P_{1}, P_{2}, B_{1}, B_{2}$ are nonnegative continuous solutions of (2.6)-(2.8) on $[0, T]$ and if $\rho_{1}, \rho_{2}$ are defined by (2.2)-(2.4) on $\mathbf{R}^{+} \times[0, T]$ then such $\rho_{1}, \rho_{2}$ provide a solution of (1.1)-(1.6) on $\mathbf{R}^{+} \times[0, T]$.

The following a priori estimates are useful to prove our existence theorem below. It will immediately follow from the bounds for $f_{i}$ and $b_{i}$ that

$$
\begin{aligned}
& M_{i}(a, t, \mathbf{P}) \leq \exp \left[-d_{i}^{*}(t-a)\right] \leq 1, \text { if } t \geq a, \\
& L_{i}(a, t, \mathbb{P}) \leq \exp \left[-d_{i}^{*} t\right] .
\end{aligned}
$$

From (2.7)-(2.8) we derive that

$$
B_{i}(t) \leq \int_{0}^{t} B_{i}^{* B}(\alpha) \exp \left[-d_{i}^{*}(t-a)\right] d a+\beta_{i}^{*} \Phi_{i}(0) \exp \left[-d_{i}^{*} t\right], i=1,2,
$$

and hence by Gronwall's inequality,

$$
B_{i}(t) \leq \beta_{i}^{*} \Phi_{i}(0) \exp \left[\delta_{i} t\right], \quad \delta_{i}=\beta_{i}^{*}-d_{i}^{*} .
$$

$(2.5),(2.6)$ and $(2.10)$ lead to 
(2.11) $\left|P_{i}(t)-\Phi_{i}(0)\right| \leq \int_{0}^{t} \beta_{i}^{*} \Phi_{i}(0) \exp \left[\delta_{i} a-d_{i}^{*}(t-a)\right] d a$

$$
\begin{aligned}
& +\int_{0}^{\infty} \varphi_{i}(a, 0)\left|1-\exp \left(-d_{i}^{*} t\right)\right| d a \\
\leq & \beta_{i}^{*} \Phi_{i}(0) \exp \left(\beta_{i}^{*} t\right)\left\{\frac{1-\exp \left[-\left(d_{i}^{*}+\beta_{i}^{*}\right) t\right]}{d_{i}^{*}+\beta_{i}^{*}}\right\}+\Phi_{i}(0) d_{i}^{* t} \\
\leq & {\left[\beta_{i}^{*} \Phi_{i}(0) \exp \left(\beta_{i}^{*} t\right)+\Phi_{i}(0) d_{i}^{*}\right] t \text { for } t>0 . }
\end{aligned}
$$

We can now prove the following result on the existence and uniqueness of solutions of the integral equations (2.6)-(2.8).

THEOREM 2. Assume that the system (1.1)-(1.6) satisfies the hypotheses $\mathrm{A}_{1}, \mathrm{~A}_{2}, \mathrm{~A}_{3}$. Then there exists a unique set of continuous functions $\left(P_{1}, P_{2}, B_{1}, B_{2}\right)$ such that

$$
P_{i}: \mathbf{R}^{+}+\mathbf{R}^{+}, \quad B_{i}: \mathbf{R}^{+}+\mathbf{R}^{+}(i=1,2),
$$

which satisfy the integral equations (2.6)-(2.8).

Proof. We will first show the existence of local solutions on some (possibly small) interval $[0, T] \subset \mathbf{R}^{+}$such that

$$
P_{i} \in C\left([0, T], \mathbf{R}^{+}\right), B_{i} \in C\left([0, T], \mathbf{R}^{+}\right), \quad i=1,2,
$$

satisfying (2.6)-(2.8) and then show the $P_{i}$ and $B_{i}$ can be continued as solutions for any finite interval in $\mathbf{R}^{+}$.

For some fixed positive number $T$ we let

$$
C^{+}[-\tau, T]=\{f \in C([-\tau, T], R) \mid f \geq 0\} \text {. }
$$

Let $\|\cdot\|_{T}$ denote the supremum norm in $C^{+}[-\tau, T]$. Then for any $(x, y) \in C^{+}[-\tau, T] \times C^{+}[-\tau, T]$ we define

$$
\begin{gathered}
\|(x, y)\|=\|x\|_{T}+\|y\|_{T}, \\
C_{T}=C^{+}[-\tau, T], \quad C_{T}^{2}=C^{+}[-\tau, T] \times C^{+}[-\tau, T] .
\end{gathered}
$$

Members of $C_{T}$ will be denoted by $z_{t}$ with the meaning that $z(t+s)=z_{t}(s)$ for $s \in[-\tau, 0]$ and $t \in[0, T]$. Now for any fixed 
$P_{t}=\left(P_{1 t}, P_{2 t}\right) \in C_{T}^{2},(2.8)$ is a linear system of uncoupled Volterra integral equations in $B=\left(B_{1}, B_{2}\right)$ and hence (2.8) will have a unique continuous solution under the assumptions $A_{1}-A_{3}$. Let us denote such a solution by

$$
B(t)=B^{T}\left(P_{t}\right), \quad t \in[0, T]
$$

or

$$
B_{1}(t)=B_{1}^{T}\left(P_{1 t}, P_{2 t}\right), B_{2}(t)=B_{2}^{T}\left(P_{1 t}, P_{2 t}\right), t \in[0, T] .
$$

If we supply this solution $\left(B_{1}, B_{2}\right)$ in (2.6) we find that (2.6) will be satisfied if and only if $\left(P_{1 t}, P_{2 t}\right)$ is a fixed point of the operator

$$
\Pi\left(P_{t}\right)=\left\{\Pi_{1}\left(P_{1 t}, P_{2 t}\right), \Pi_{2}\left(P_{1 t}, P_{2 t}\right)\right\}
$$

where $\Pi_{1}$ and $\Pi_{2}$ respectively denote the right sides of (2.6) for $i=1,2$. It is not difficult to see from the nature of $M_{i}, L_{i}$ and the hypotheses on $f_{i}, b_{i}, \varphi_{i}$ that

$$
B: C_{T}^{2} \rightarrow C_{T}^{2} \text { and } \Pi: C_{T}^{2}+C_{T}^{2}
$$

(which guarantees the nonnegativity of $P_{i}$ and $B_{i}$ ). We will first show that there exist positive constants $r^{*}, t^{*}$ such that $\Pi$ is a mapping of a sphere

$$
S_{t^{*}}\left(\Phi, r^{*}\right)=\left\{P_{t}=\left(P_{1 t}, P_{2 t}\right) \in C_{t^{*}}^{2} \mid\left\|P_{t}-\Phi_{t}\right\|_{t^{*}} \leq r^{*}\right\}
$$

into itself and also is a contraction where $\Phi_{t}$ is defined on $\left[-\tau, t^{*}\right]$ by the following:

$$
\begin{aligned}
\Phi_{t} & =\left(\Phi_{1 t}, \Phi_{2 t}\right) ; \\
\Phi_{i t} & = \begin{cases}\int_{0}^{\infty} \varphi_{i}(a, s) d a & \text { for } s \in[-\tau, 0], \\
\int_{0}^{\infty} \varphi_{i}(a, 0) d a & \text { for } s \in\left[0, t^{*}\right] .\end{cases}
\end{aligned}
$$


We note that similar to (2.11) one gets the estimates

$$
\left|\Pi_{i}(P)(t)-\Phi_{i}(0)\right| \leq\left\{\beta_{i}^{* \Phi} \Phi_{i}(0) \exp \left[\beta_{i}^{*} t\right]+\Phi_{i}(0) d_{i}^{*}\right\} t
$$

which together with $(2.7)$ and $(2.12)$ lead to

$$
\left\|\Pi\left(P_{t}\right)-\Phi_{t}\right\|_{t^{*}} \leq\left(\sum_{j=1}^{2}\left\|\Phi_{j t}\right\|_{t^{*}}\left[\beta_{j}^{*} \exp \left(\beta_{j}^{*} t\right)+d_{i}^{*}\right]\right) t^{*} .
$$

Thus if we choose

$$
r^{*}=T \sum_{j=1}^{2}\left\|\Phi_{j t}\right\|_{t}\left[\beta_{j}^{*} \exp \left(\beta_{j}^{*} T\right)+d_{j}^{*}\right]
$$

then $\Pi$ is a mapping of $S_{t^{*}}\left(\Phi, r^{*}\right)$ into itself for all $t^{*} \leq T$. We then will have to show that for small $t^{*}>0, \Pi$ is a contraction.

Let $P_{t}^{(1)}$ and $P_{t}^{(2)}$ be arbitrarily chosen in $S_{t^{*}}\left(\Phi, r^{*}\right)$ and consider $\left|\Pi_{1}\left(P_{t}^{(1)}\right)-\Pi_{1}\left(P_{t}^{(2)}\right)\right|$ for $t \leq t^{*}$. Corresponding to the chosen $P_{t}^{(1)}, P_{t}^{(2)}$ we let $B^{(1)}(a)=B\left(P_{t}^{(1)}\right)$ and $B^{(2)}(a)=B\left(P_{t}^{(2)}\right)$. Then from $(2.6)$,

$$
\begin{aligned}
\left|\Pi_{1}\left(P^{(1)}(t)\right)-\Pi_{1}\left(P^{(2)}(t)\right)\right| & \leq \mid \int_{0}^{t} B_{1}^{(1)}(a)\left\{M_{1}\left(a, t, P_{t}^{(1)},-M_{1}\left(a, t, P_{t}^{(2)}\right)\right\} d a \mid\right. \\
& +\left|\int_{0}^{t}\left[B_{1}^{(1)}(a)-B_{2}^{(2)}(a)\right] M_{1}\left(a, t, P_{t}^{(2)}\right) d a\right| \\
& +\mid \int_{0}^{t} \varphi_{1}(a, 0)\left[L_{1}\left(a, t, P_{t}^{(1)}\right)-L_{1}\left(a, t, P_{t}^{(2)}\right] d a \mid\right. \\
& =J_{1}+J_{2}+J_{3} \text { (say). }
\end{aligned}
$$

Using the elementary inequality

$$
\left|e^{x}-e^{y}\right| \leq|x-y| \text { for } x, y \leq 0
$$

we derive from (2.5) that 
Two species competition

281

$$
\begin{aligned}
\left|M_{1}\left(a, t, P_{t}^{(1)}\right)-M_{1}\left(a, t, P_{t}^{(2)}\right)\right| & \\
& \leq \int_{0}^{t-a}\left|f_{1}\left(s, P_{t}^{(1)}(a+s)\right)-f_{1}\left(s, P_{t}^{(2)}(a+s)\right)\right| d s \\
& \left.\leq d_{1}\left\|P_{t}^{(1)}-P_{t}^{(2)}\right\|_{t^{*}} \text { (using } A_{1}\right) .
\end{aligned}
$$

Now, using (2.10),

$$
\begin{aligned}
\left|J_{1}\right| & \leq \int_{0}^{t} \beta_{i}^{* \Phi_{1}}(0) e^{\delta_{1} a}\left(\int_{0}^{t-a} d_{1}\left\|P_{t}^{(1)} P_{t}^{(2)}\right\|_{t^{*}} d s\right) d a \\
& \leq d_{1} \beta_{1}^{* \Phi_{1}}(0) t^{2}\left\|P_{t}^{(1)}-P_{t}^{(2)}\right\| e^{\delta_{1} t} / 2 .
\end{aligned}
$$

In a similar way

$$
\begin{aligned}
\left|J_{3}\right| & \leq \int_{0}^{\infty} \varphi_{1}(a, 0)\left[\int_{0}^{t}\left|f_{1}\left(a+s, P_{t}^{(1)}(s)\right)-f_{1}\left(a+s, P_{t}^{(2)}(s)\right)\right| d s\right] d a \\
& \leq d_{1} \Phi_{1}(0)\left\|P_{t}^{(1)}-P_{t}^{(2)}\right\|_{t^{*}} t .
\end{aligned}
$$

Now we have to estimate $\left|B_{1}^{(1)}(a)-B_{1}^{(2)}(a)\right|$ in order to estimate $J_{2}$; from (2.8),

$$
\begin{aligned}
& B_{1}^{(1)}(a)-B_{1}^{(2)}(a) \\
& =\int_{0}^{a}\left[_{1}^{(1)}(\sigma)-B_{1}^{(2)}(\sigma)\right] b_{1}\left(a-\sigma, P_{t}^{(1)}(a)\right) M_{1}\left(\sigma, a, P_{t}^{(1)}(a)\right) d \sigma+\int_{0}^{a} B_{1}^{(2)}(\sigma) \\
& \quad \times\left[b_{1}\left(a-\sigma, P_{t}^{(1)}(a)\right) M_{1}\left(\sigma, a, P_{t}^{(1)}(a)\right)-b_{1}\left(a-\sigma, P_{t}^{(2)}(a)\right) M_{1}\left(\sigma, a, P_{t}^{(2)}(a)\right] d \sigma\right. \\
& \quad+\int_{0}^{\infty} \varphi_{1}(\sigma, 0)\left[b_{1}\left(\sigma+a, P_{t}^{(1)}(a)\right)-b_{1}\left(\sigma+a, P_{t}^{(2)}(a)\right)\right] L_{1}\left(\sigma, a, P_{t}^{(1)}(a)\right) d \sigma \\
& \quad+\int_{0}^{\infty} \varphi_{1}(\sigma, 0) b_{1}\left(\sigma+a, P_{t}^{(2)}(a)\right)\left[L_{1}\left(\sigma, a, P_{t}^{(1)}(a)\right)-L_{1}\left(\sigma, a, P_{t}^{(2)}(a)\right)\right] d \sigma \\
& =R_{1}+R_{2}+R_{3}+R_{4} \text { (say). }
\end{aligned}
$$

By the uniform Lipschitz continuity of $b_{1}$ in $A_{2}$ we get

$$
\left|b_{1}\left(a+\sigma, P_{t}^{(1)}(a)\right)-b_{1}\left(\sigma+a, P_{t}^{(2)}(a)\right)\right| \leq \beta_{1}\left\|P_{t}^{(1)}-P_{t}^{(2)}\right\|_{t^{*}} \text { for } a \in\left[0, t^{*}\right]
$$

https://doi.org/10.1017/S0004972700005281 Published online by Cambridge University Press 
and hence we have

$$
\begin{aligned}
& \left|R_{3}(a)+R_{4}(a)\right| \leq \beta_{1} \Phi_{1}(0)\left\|P_{t}^{(1)}-P_{t}^{(2)}\right\|_{t^{*}}+\Phi_{1}(0) \beta_{1} d_{1}\left\|P_{t}^{(1)}-P_{t}^{(2)}\right\|_{t^{*}} a, \\
& \left|R_{2}(a)\right|=\left[\int _ { 0 } ^ { a } \left[B_{1}^{(2)}(\sigma)\left\{b_{1}\left(a-\sigma, P_{t}^{(1)}(a)\right)-b_{1}\left(a-\sigma, P_{t}^{(2)}(a)\right)\right\} M_{1}\left(\sigma, a, P_{t}^{(1)}(a)\right)\right.\right. \\
& \left.\left.+b_{1}\left(a-\sigma, P_{t}^{(2)}(a)\right)\left\{M_{1}\left(\sigma, a, P_{t}^{(1)}(a)\right)-M_{1}\left(\sigma, a, P_{t}^{(2)}(a)\right)\right\}\right] d \sigma\right]
\end{aligned}
$$

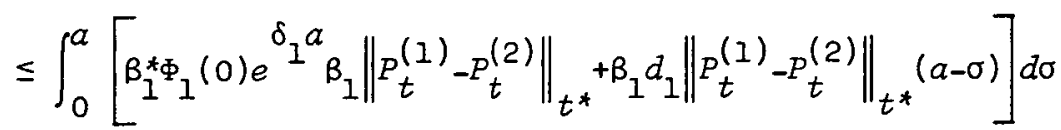

$$
\begin{aligned}
& \leq \beta_{1}^{* \Phi_{1}}(0) e^{\delta_{1} a_{1}}\left\|P_{t}^{(1)}{ }_{-P_{t}^{(2)}}^{(2)}\right\|_{t *}\left\{\beta_{1} a+d_{1} a^{2} / 2\right\} \text {. }
\end{aligned}
$$

Thus

$$
\begin{aligned}
\left|B_{1}^{(1)}(a)-B_{1}^{(2)}(a)\right| & =\int_{0}^{a}\left|B_{1}^{(1)}(\sigma)-B_{1}^{(2)}(\sigma)\right| \beta_{1}^{*} d \sigma \\
& \quad+\Phi_{1}(0)\left\|P_{t}^{(1)}-P_{t}^{(2)}\right\|_{t^{*}}\left\{\beta_{1}+\beta_{2} d_{1} a+\beta_{1}^{*}\left(\beta_{2} a+d_{1} a^{2} / 2\right) e^{\delta_{1} a}\right\} \\
& \leq B_{1}^{*} \int_{0}^{a}\left|B_{1}^{(1)}(\sigma)-B_{1}^{(2)}(\sigma)\right| d \sigma+\left(r^{*}+\left\|\Phi_{t}\right\|_{t *}\right)\left\|P_{t}^{(1)} P_{t}^{(2)}\right\|_{t^{*}} g_{1}(a)
\end{aligned}
$$

where $g_{1}(a) \rightarrow B_{1}$ as $a \rightarrow 0$. By Gronwall's inequality it will follow that

$$
\left|B_{1}^{(1)}(a)-B_{1}^{(2)}(a)\right| \leq\left(r^{*}+\left\|\Phi_{t^{*}}\right\|_{t^{*}}\right)\left\|P_{t}^{(1)}-P_{t}^{(2)}\right\|_{t^{*}}\left\{g_{1}(a)+O(a)\right\} .
$$

Using this estimate for $R_{1}(a)$ we get

$$
\begin{aligned}
\left|R_{1}(a)\right| & \leq\left|\int_{0}^{a}\left[B_{1}^{(1)}(\sigma)-B_{1}^{(2)}(\sigma)\right] b_{1}\left(a-\sigma, P_{t}^{(1)}(a)\right) M_{1}\left(\sigma, a, P_{t}^{(1)}(a)\right) d \sigma\right| \\
& \leq B_{1}^{*}\left(r^{*}+\left\|\Phi_{t}\right\|_{t^{*}}\right)\left\|P_{t}^{(1)}-P_{t}^{(2)}\right\|_{t^{*}} O(a) \text { as } a \rightarrow 0 .
\end{aligned}
$$

Thus 


$$
\left|B_{1}^{(1)}(a)-B_{1}^{(2)}(a)\right| \leq\left(r^{*}+\left\|\Phi_{t}\right\|_{t^{*}}\right)\left\|P_{t}^{(1)}-P_{t}^{(2)}\right\|_{t^{*}} O(1) \text { as } a \rightarrow 0
$$

and hence

$$
\left|\Pi_{1}\left(P_{t}^{(1)}\right)-\Pi_{1}\left(P_{t}^{(2)}\right)\right| \leq\left(r^{*}+\left\|\Phi_{t}\right\|_{t^{*}}\right)\left\|P_{t}^{(1)}-P_{t}^{(2)}\right\|_{t^{*}} O(t) \text { as } \quad t \rightarrow 0
$$

which together with a similar bound for $\left|\Pi_{2}\left(P_{t}^{(1)}\right)-\Pi_{2}\left(P_{t}^{(2)}\right)\right|$ leads to

$$
\left\|\Pi\left(P_{t}^{(1)}\right)-\Pi\left(P_{t}^{(2)}\right)\right\| \leq\left(r^{*}+\left\|\Phi_{t}\right\|_{t^{*}}\right)\left\|P_{t}^{(1)}-P_{t}^{(2)}\right\|_{t^{*}} O\left(t^{*}\right)
$$

as $t^{*} \rightarrow 0$ showing that if $t^{*}$ is small enough, the mapping $I$ on $S_{t^{*}}\left(\Phi, r^{*}\right)$ is a contraction. Hence the system $(2.6)-(2.8)$ has a unique solution for $0 \leq t \leq t^{*}$ for $t^{*}$ sufficiently small and positive.

Now if $[0, T]$ is any finite interval on $\mathbf{R}^{+}$, we can consider $[O, T]$ as a finite union of intervals of length less than or equal to $t^{*}$. Since the estimate (2.11) is valid for any finite interval we can extend the solution of $(2.6)-(2.8)$ from $\left[0, t^{*}\right]$ to $[0, T]$ and this completes the proof.

\section{Stationary age distributions and their linear stability}

We will now establish the existence of time independent solutions $\rho_{1}^{*}, \rho_{2}^{*}$ of $(1.1)-(1.6) ;$ such solutions satisfy

$$
\begin{gathered}
\frac{d \rho_{i}^{*}(a)}{d a}+f_{i}\left(a, P_{1}^{*}, P_{2}^{*}\right) \rho_{i}^{*}(a)=0, i=1,2, a>0, \\
P_{i}^{*}=\int_{0}^{\infty} \rho_{i}^{*}(a) d a, i=1,2, \\
\rho_{i}^{*}(0)=\int_{0}^{\infty} b_{i}\left(a, P_{1}^{*}, P_{2}^{*}\right) \rho_{i}^{*}(a) d a, i=1,2 .
\end{gathered}
$$

All solutions of (3.1) are of the form

(3.4) $\rho_{i}^{*}(a)=\rho_{i}^{*}(0) \exp \left[-\int_{0}^{a} f_{i}\left(s, P_{1}^{*}, P_{2}^{*}\right) d s\right], i=1,2, a \geq 0$,

which together with (3.3) lead to 


$$
\begin{aligned}
& 1=\int_{0}^{\infty} b_{1}\left(a, P_{1}^{*}, P_{2}^{*}\right) \exp \left[-\int_{0}^{a} f_{1}\left(s, P_{1}^{*}, P_{2}^{*}\right) d s\right] d a, \\
& 1=\int_{0}^{\infty} b_{2}\left(a, P_{1}^{*}, P_{2}^{*}\right) \exp \left[-\int_{0}^{a} f_{2}\left(s, P_{1}^{*}, P_{2}^{*}\right) d s\right] d a .
\end{aligned}
$$

Thus the existence of stationary solutions of $(1.1)-(1.6)$ reduces to the existence of a pair of positive constants $P_{1}^{*}, P_{2}^{*}$ satisfying (3.5). We can now prove the following.

THEOREM 3. Assume that $f_{i}$ and $b_{i}$ satisfy $\left(\mathrm{A}_{1}\right)$ and $\left(\mathrm{A}_{2}\right)$ and let

$$
\begin{aligned}
& \text { (3.6) } F_{i}\left(P_{1}, P_{2}\right)=\int_{0}^{\infty} b_{i}\left(a, P_{1}, P_{2}\right) \exp \left[-\int_{0}^{a} f_{i}\left(s, P_{1}, P_{2}\right) d s\right] d a \text {, } \\
& i=1,2, P_{1} \geq 0, P_{2} \geq 0,
\end{aligned}
$$

and suppose the following hold:

(3.7) $\mathrm{A}_{4}, F_{i}(0,0)>1, i=1,2$;

A5. there exists a positive constant $C$ for which

$$
\begin{aligned}
& F_{1}(C, 0)<F_{2}(C, 0), \\
& F_{1}(0, C)^{\prime}>F_{2}(0, C) .
\end{aligned}
$$

Then there exists a unique pair $\left(P_{1}^{*}, P_{2}^{*}\right)$ of real numbers $P_{1}^{*}>-0$, $P_{2}^{*}>0$ such that

$$
F_{1}\left(P_{1}^{*}, P_{2}^{*}\right)=1=F_{2}\left(P_{1}^{*}, P_{2}^{*}\right)
$$

and the unique nonnegative solution of (3.1)-(3.3) is given by

$$
\begin{array}{r}
\rho_{i}^{*}(a)=\left(P_{i}^{*} \exp \left[-\int_{0}^{a} f_{i}\left(s, P_{1}^{*}, P_{2}^{*}\right) d s\right]\right) /\left[\int_{0}^{\infty} \exp \left[-\int_{0}^{a} f_{i}\left(s, P_{1}^{*}, P_{2}^{*}\right) d s\right] d a\right], \\
i=1,2, a \geq 0 .
\end{array}
$$

Proof. Consider the elements of the surfaces defined by

$$
\boldsymbol{z}=F_{1}\left(P_{1}, P_{2}\right), \boldsymbol{z}=F_{2}\left(P_{1}, P_{2}\right), P_{1} \geq 0, P_{2} \geq 0,
$$


in the $\left(P_{1}, P_{2}, z\right)$ space. By hypothesis $\left(A_{1}\right)$ we have $\partial F_{i} / \partial P_{j}<0$ and, by $\left(A_{2}\right), \partial b_{i} / \partial P_{j} \leq 0$ for all $P_{1} \geq 0, P_{2} \geq 0, i=1,2$,

$j=1,2$. Hence by $\left(\mathrm{A}_{4}\right)$ the intersections of these surfaces with the plane $z=1$ define two curves on the $z=1$ plane connecting the lines $\left\{z=1, P_{1}=0, P_{2} \geq 0\right\}$ and $\left\{z=1, P_{2}=0, P_{1} \geq 0\right\}$. These two curves are defined by

$$
F_{1}\left(P_{1}, P_{2}\right)=1 \text { and } F_{2}\left(P_{1}, P_{2}\right)=1, P_{1}, P_{2} \geq 0 \text {, }
$$

which by $\left(A_{5}\right)$ will intersect at a unique point say $\left(P_{1}^{*}, P_{2}^{*}\right)$ with $P_{1}^{*}>0, P_{2}^{*}>0$ (see figure). The uniqueness of the point $\left(P_{1}^{*}, P_{2}^{*}\right)$ is a consequence of the smoothness of the surfaces $z=F_{1}\left(P_{1}, P_{2}\right)$ and $z=F_{2}\left(P_{1}, P_{2}\right)$.

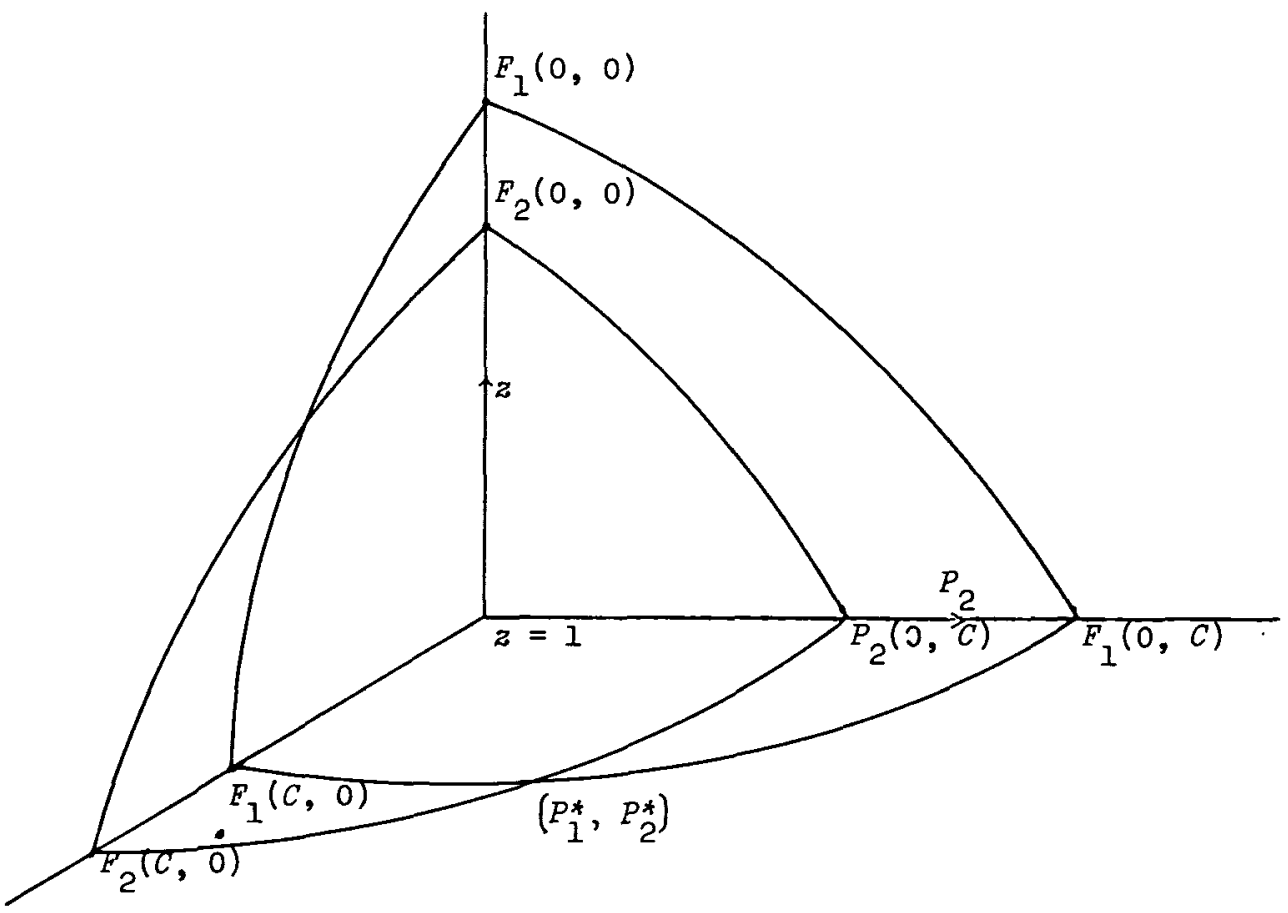

(The figure corresponds to the case $F_{1}(0,0)>F_{2}(0,0)>1$. The other possibility $1<F_{1}(0,0) \leq F_{2}(0,0)$ is treated similarly.) It will now 
follow that for such a pair $\left(P_{1}^{*}, P_{2}^{*}\right)$ the unique nonnegative solution of $(3.1)-(3.3)$ is given by $(3.10)$.

Now to examine the linear stability of the stationary age distributions $\rho_{1}^{*}, \rho_{2}^{*}$ we let

$$
\left\{\begin{aligned}
\rho_{i}(a, t) & =\rho_{i}^{*}(a)+u_{i}(a, t), \\
P_{i}(t) & =P_{i}^{*}+p_{i}(t), i=1,2, a>0, t>0, \\
p_{i}(t) & =\int_{0}^{\infty} u_{i}(a, t) d a,
\end{aligned}\right.
$$

in (1.1)-(1.5) and derive the following yariational system after neglecting the nonlinear terms in the perturbations $u_{i}$ and $p_{i}(i=1,2)$. (Such a procedure of linear stability analysis can be justified as has been done in Gurtin and MacCamy [4].)

$$
\left\{\begin{array}{l}
D u_{i}(a, t)=-f_{i}\left(a, P_{1}^{*}, P_{2}^{*}\right) u_{i}(a, t)-\rho_{i}^{*}(a)\left\{\sum_{i=1}^{2} \frac{\partial f_{i}}{\partial P_{j}^{*}} p_{i j}(t)\right\}, \\
u_{i}(0, t)=\int_{0}^{\infty} b_{i}\left(a, P_{1}^{*}, P_{2}^{*}\right) u_{i}(a, t) d a+\int_{0}^{\infty} \rho_{i}^{*}(a)\left\{\sum_{j=1}^{2} \frac{\partial b}{\partial P_{j}^{*}} p_{i j}(t)\right\} d a
\end{array}\right.
$$

where $i=1,2$ and

$$
\begin{array}{ll}
p_{11}(t)=p_{1}(t), & p_{12}(t)=p_{2}(t-\tau), \\
p_{21}(t)=p_{1}(t-\tau), & p_{22}(t)=p_{2}(t) .
\end{array}
$$

To consider the asymptotic behaviour as $t \rightarrow \infty$ of solutions of (3.12) we let

$$
\left\{\begin{aligned}
u_{i}(a, t) & =\xi_{i}(a) \exp [\lambda t], \quad \xi_{i}(a) \rightarrow 0 \text { as } a \rightarrow \infty \\
p_{i}(t) & =p_{i}^{*} \exp [\lambda t], \\
p_{i}^{*} & =\int_{0}^{\infty} \xi_{i}(a) d a .1,2, a>0, t>0,
\end{aligned}\right.
$$

Using (3.14) in (3.12)-(3.13), 


$$
\begin{gathered}
\frac{d \xi_{i}}{d a}+\lambda \xi_{i}=-f_{i}\left(a, P_{1}^{*}, P_{2}^{*}\right) \xi_{i}(a)-\rho_{i}^{*}(a)\left\{\sum_{j=1}^{2} \frac{\partial f_{i}}{\partial P_{j}^{*}} p_{i j}^{*}\right\}, \\
\xi_{i}(0)=\int_{0}^{\infty} b_{i}\left(a, P_{1}^{*}, P_{2}^{*}\right) \xi_{i}(a) d a+\int_{0}^{\infty} \rho_{i}^{*}(a)\left\{\sum_{j=1}^{2} \frac{\partial b_{i}}{\partial P_{j}^{*}} p_{i j}^{*}\right\} d a,
\end{gathered}
$$

where $i=1,2$, and

$$
\begin{aligned}
& p_{11}^{*}=p_{1}^{*}, p_{12}^{*}=p_{2}^{*} \exp \{-\lambda \tau\}, \\
& p_{21}^{*}=p_{1}^{*} \exp \{-\lambda \tau\}, p_{22}^{*}=p_{2}^{*} .
\end{aligned}
$$

If we integrate the first of (3.15) and use the fact that $\xi_{i}(a) \rightarrow 0$ as $a+\infty$ we can derive that $p_{1}^{*}$ and $p_{2}^{*}$ are governed by

$$
\text { (3.16) } \begin{aligned}
\lambda p_{1}^{*}-\int_{0}^{\infty} \rho_{1}^{*}(\alpha)\left[\left(\frac{\partial b_{1}}{\partial P_{1}^{*}}-\frac{\partial f_{1}}{\partial P_{1}^{*}}\right) p_{1}^{*}+\left(\frac{\partial b_{1}}{\partial P_{2}^{*}}-\frac{\partial f_{1}}{\partial P_{2}^{*}}\right) p_{2}^{*} e^{-\lambda \tau}\right] d a \\
=\int_{0}^{\infty}\left[b_{1}\left(a, P_{1}^{*}, P_{2}^{*}\right)-f_{1}\left(a, P_{1}^{*}, P_{2}^{*}\right)\right] \xi_{1}(a) d a,
\end{aligned}
$$$$
\text { (3.17) } \lambda p_{2}^{*}-\int_{0}^{\infty} \rho_{2}(a)\left[\left(\frac{\partial b_{2}}{\partial P_{1}^{*}}-\frac{\partial f_{2}}{\partial P_{1}^{*}}\right) p_{1}^{*} e^{-\lambda \tau}+\left(\frac{\partial b_{2}}{\partial P_{2}^{*}}-\frac{\partial f_{2}}{\partial P_{2}^{*}}\right) p_{2}^{*}\right] d a
$$$$
=\int_{0}^{\infty}\left[b_{2}\left(a, P_{1}^{*}, P_{2}^{*}\right)-f_{2}\left(a, P_{1}^{*}, P_{2}^{*}\right)\right] \xi_{2}(a) d a \text {. }
$$

From the first of (3.15) we get

$$
\begin{aligned}
\xi_{i}(a)=\left\{\xi_{i}(0) \rho_{i}^{*}(a) / \rho_{i}^{*}(0) \exp [\lambda a]\right\} & \\
& -\rho_{i}^{*}(a) \exp [-\lambda a] \int_{0}^{a}\left\{\sum_{j=1}^{2} \frac{\partial f_{i}}{\partial P_{j}^{*}} p_{i j}^{*}\right\} e^{\lambda s} d s, i=1,2,
\end{aligned}
$$

which by the second of (3.15) becomes,

$$
\text { (3.19) } \begin{aligned}
\xi_{i}(a)=\left[\rho_{i}^{*}(a) / \rho_{i}^{*}(0) c_{i} \exp (\lambda a)\right]\left[\int_{0}^{\infty} \rho_{i}^{*}(a)\left\{\sum_{j=1}^{2} \frac{\partial b_{i}}{\partial P_{j}^{*}} p_{i j}^{*}\right\} d a\right. \\
\left.-\int_{0}^{\infty} b_{i}\left(a, P_{1}^{*}, P_{2}^{*}\right) \rho_{i}^{*}(a) e^{-\lambda a}\left\{\int_{0}^{a}\left[\sum_{j=1}^{2} \frac{\partial f_{i}}{\partial P_{j}^{*}} p_{i j}^{*}\right\} e^{\lambda s} d s\right\} d a\right] \\
-\rho_{i}^{*}(a) e^{-\lambda a} \int_{0}^{a}\left\{\sum_{j=1}^{2} \frac{\partial f_{i}}{\partial P_{j}^{*}} p_{i j}^{*}\right\} e^{\lambda s} d s, i=1,2,
\end{aligned}
$$


where

$$
c_{i}=1-\frac{1}{\rho_{i}^{*}(0)} \int_{0}^{\infty} b_{i}\left(a, P_{1}^{*}, P_{2}^{*}\right) \rho_{i}^{*}(a) e^{-\lambda a} d a, i=1,2 .
$$

Now supplying $\xi_{1}(a)$ and $\xi_{2}(a)$ from (3.19) in (3.16)-(3.17) we derive that

$$
\left(\lambda-A_{11}\right) p_{1}^{*}-A_{12} p_{2}^{*} \exp (-\lambda \tau)=g_{11}(\lambda) p_{1}^{*}+g_{12}(\lambda) p_{2}^{*} \exp (-\lambda \tau),
$$

$$
-A_{21} p_{1}^{*} \exp (-\lambda \tau)+\left(\lambda-A_{22}\right) p_{2}^{*}=g_{21}(\lambda) p_{1}^{*} \exp (-\lambda \tau)+g_{22}(\lambda) p_{2}^{*},
$$

where

$$
A_{i j}=\int_{0}^{\infty} \rho_{i}^{*}(a)\left[\frac{\partial b}{\partial P_{j}^{*}}-\frac{\partial f_{i}}{\partial P_{j}^{*}}\right] d a,
$$

$$
\begin{aligned}
g_{i j}(\lambda)= & {\left[\rho_{i}^{*}(0) c_{i}\right]^{-1}\left\{\int_{0}^{\infty} \sigma_{i}(a) \rho_{i}^{*}(a) e^{-\lambda a} d a\right\} } \\
& \times\left\{\int_{0}^{\infty}\left[\rho_{i}^{*}(a) \frac{\partial b_{i}}{\partial P_{j}^{*}}-b_{i}\left(a, P_{1}^{*}, P_{2}^{*}\right) \rho_{i}^{*}(a) e^{-\lambda a}\left(\int_{0}^{a} \frac{\partial f_{i}}{\partial P_{j}^{*}} e^{\lambda s} d s\right)\right] d a\right\} \\
& -\int_{0}^{\infty} \sigma_{i}(a) \rho_{i}^{*}(a) e^{-\lambda a}\left(\int_{0}^{a} \frac{\partial f_{i}}{\partial P_{j}^{*}} e^{\lambda s} d s\right) d a, i, j=1,2,
\end{aligned}
$$

$$
\sigma_{i}(a)=b_{i}\left(a, P_{1}^{*}, P_{2}^{*}\right)-f_{i}\left(a, P_{1}^{*}, P_{2}^{*}\right), i=1,2 .
$$

To solve the linear perturbational system (3.12) it is enough to find the constants $\lambda, p_{1}^{*}, p_{2}^{*}$ satisfying (3.21) since one can then use (3.19) to find the perturbations from (3.14). A nontrivial solution $\left(p_{1}^{*}, p_{2}^{*}\right)$ for (3.21) will exist if and only if $\lambda$ is a root of the equation

$$
\operatorname{det}\left[\begin{array}{cc}
\lambda-\left(A_{11}+g_{11}(\lambda)\right) & -\left(A_{12}+g_{12}\right) e^{-\lambda \tau} \\
-\left(A_{21}+g_{21}\right) e^{-\lambda \tau} & \lambda-\left(A_{22}+g_{22}(\lambda)\right)
\end{array}\right]=0
$$

or equivalently

$$
\text { (3.25) } \lambda^{2}-\lambda\left(A_{11}+A_{22}\right)+A_{11} A_{22}-A_{12} A_{21} \exp [-2 \lambda \tau]=\lambda S_{1}(\lambda)+S_{2}(\lambda, \tau)
$$

where 


$$
\begin{aligned}
S_{1}(\lambda) & =g_{11}(\lambda)+g_{22}(\lambda) \\
S_{2}(\lambda, \tau)= & {\left[A_{21} g_{12}(\lambda)+A_{12} g_{21}(\lambda)+g_{12}(\lambda) g_{21}(\lambda)\right] \exp [-2 \lambda \tau] } \\
& -A_{11} g_{22}(\lambda)-A_{22} g_{11}(\lambda)-g_{11}(\lambda) g_{22}(\lambda) .
\end{aligned}
$$

The following result establishes the linear local asymptotic stability of the stationary age distributions $\rho_{1}^{*}(a)$ and $\rho_{2}^{*}(a)$.

THEOREM 4. Assume the following:

A6. $0<\beta_{i}^{*}<d_{i}^{*}(i=1,2)\left(\operatorname{see}\left(A_{1}\right)\right.$ and $\left.\left(A_{2}\right)\right)$;

$$
\text { A7. } \begin{aligned}
\left\{A_{11}+A_{22}+\left\|S_{1}(0)\right\|\right\}^{2}<4\left\{\left(A_{11} A_{22}-A_{12} A_{21}\right)-\left\|S_{2}(0)\right\|\right\}, \\
\begin{aligned}
\left\|S_{1}(0)\right\| & =\left|g_{11}(0)\right|+\left|g_{22}(0)\right|, \\
\left\|S_{2}(0)\right\|= & \left|A_{11} g_{12}(0)\right|+\left|A_{12} g_{21}(0)\right|+\left|g_{12}(0) g_{21}(0)\right| \\
& +\left|A_{11} g_{22}(0)\right|+\left|A_{22} g_{11}(0)\right|+\left|g_{11}(0) g_{22}(0)\right| .
\end{aligned}
\end{aligned}
$$

Then all the roots of (3.25) have negative real parts and hence the perturbations $u_{i}(a, t)=\xi_{i}(a) \exp [\lambda t] \rightarrow 0$ as $t \rightarrow \infty$ for $i=1,2$ and $a \geq 0$.

Proof. Define $F_{1}(\lambda, \tau)$ and $F_{2}(\lambda, \tau)$ as follows:

$$
\begin{aligned}
& F_{1}(\lambda, \tau)=\lambda^{2}-\lambda\left(A_{11}+A_{22}\right)+A_{11} A_{22}-A_{12} A_{21} \exp (-2 \lambda \tau) ; \\
& F_{2}(\lambda, \tau)=\lambda S_{1}(\lambda)+S_{2}(\lambda, \tau) .
\end{aligned}
$$

Using $\left(A_{6}\right)$ one can show that for $\lambda$ with $\operatorname{Re}(\lambda) \geq 0, c_{1}$ and $c_{2}$ are positive and bounded away from zero. Now since $A_{i j}>0$ and $\tau \geq 0$ we find from the nature of the dependence of $g_{i j}$ on $\lambda$ that for all $\lambda=\mu \pm i \omega$ with $\mu \geq 0$ and $\omega \geq 0$ we have

$$
\text { (3.26) } \begin{aligned}
\mid F_{1}(\lambda, \dot{\tau}) & -F_{2}(\lambda, \tau) \mid \\
& \geq\left|F_{1}(\lambda, 0)\right|-\left|F_{2}(\lambda, \tau)\right| \\
& \geq|\lambda|^{2}-|\lambda|\left\{A_{11}+A_{22}+\left\|S_{1}(0)\right\|\right\}+\left\{A_{11} A_{22}-A_{12} A_{21}-\left\|S_{2}(0, \tau)\right\|\right\} \\
& \geq\left[(|\lambda|-p)^{2}+q\right]^{2}
\end{aligned}
$$


where

$$
\begin{aligned}
& p=\left\{A_{11}+A_{22}+\left\|S_{1}(0)\right\|\right\} / 2, \\
& q=\left\{4\left|\left(A_{11} A_{22}-A_{12} A_{21}\right)-\left\|S_{2}(0, \tau)\right\|\right|-\left|A_{11}+A_{22}+\left\|S_{1}(0)\right\|\right|^{2}\right\} / 2 .
\end{aligned}
$$

It will now follow, from (A) and (3.26), $F_{1}(\lambda, \tau)=F_{2}(\lambda, \tau)$ cannot have roots with zero or positive real parts and this completes the proof.

In conclusion we remark that the condition (3.7) means that the net reproduction rates of each species is greater than unity; a condition of this type is well known in age dependent populations. (3.8) can be interpreted to mean that the intraspecific competitive inhibition is higher than that of the interspecific interaction. The conditions of Theorem 4 are analytical and do not lend themselves for any worthwile interpretation in terms of the model parameters.

\section{References}

[1] K. Gopalsamy, "Time lags and global stability in two species competition", Bul2. Math. Biol. 42 (1980), 729-737.

[2] K. Gopalsamy, "Harmless delays in model systems", submitted.

[3] Morten E. Gurtin \& Richard C. MacCamy, "Non-linear age-dependent population dynamics", Arch. Rational Mech. Anal. 54 (1974), $281-300$.

[4] Morton E. Gurtin and Richard C. MacCamy, "Some simple models for nonlinear age-dependent population dynamics", Math. Biosci. 43 (1979), 199-211.

[5] M.E. Gurtin and R.C. MacCamy, "Population dynamics with age dependence", Nonlinear analysis and mechanics: Heriot-Watt Symposium, Vol. III, 1-35 (Research Notes in Mathematics, 30. Pitman, London, 1979).

[6] Morton E. Gurtin and Daniel S. Levine, "On predator-prey interactions with predation dependent on age of prey", Math. Biosci. 47 (1979), 207-219. 
[7] Adolf Haimovici, "On the growth of a population dependent on ages and involving resources and polluation", Math. Biosci. 43 (1979), 213-237.

[8] Manuel Rotenberg, "Equilibrium and stability in populations whose interactions are age-specific", J. Theoret. Biol. 54 (1975), $207-224$.

School of Mathematics,

Flinders University of South Australia,

Bedford Park,

South Australia 5042,

Australia. 